\title{
Looking forward to test the KOTO anomaly with FASER
}

\author{
Felix Kling $\odot^{*}$ \\ SLAC National Accelerator Laboratory, 2575 Sand Hill Road, Menlo Park, California 94025, USA \\ Sebastian Trojanowskiఠ ${ }^{\dagger}$ \\ Consortium for Fundamental Physics, School of Mathematics and Statistics, University of Sheffield, \\ Hounsfield Road, Sheffield, S3 7RH, United Kingdom \\ and National Centre for Nuclear Research, Pasteura 7, 02-093 Warsaw, Poland
}

(Received 24 June 2020; accepted 22 July 2020; published 31 July 2020)

\begin{abstract}
The search for light and long-lived particles at the LHC will be intensified in the upcoming years with a prominent role of the new FASER experiment. In this study, we discuss how FASER could independently probe such scenarios relevant for new physics searches at kaon factories. We put an emphasis on the proposed explanations for the recently observed three anomalous events in the KOTO experiment. The baseline of FASER precisely corresponds to the proposed lifetime solution to the anomaly that avoids the NA62 bounds on charged kaons. As a result, the experiment can start constraining relevant models within the first few weeks of its operation. In some cases, it can confirm a possible discovery with up to $10^{4}$ spectacular high-energy events in FASER during LHC run 3. Further complementarities between FASER and kaon factories, which employ FASER capability to study $\gamma \gamma$ signatures, are illustrated for the model with axionlike particles dominantly coupled to $S U(2)_{W}$ gauge bosons.
\end{abstract}

DOI: 10.1103/PhysRevD.102.015032

\section{INTRODUCTION}

The quest for beyond the Standard Model (BSM) physics is undoubtedly among the biggest challenges in contemporary physics. It has typically been driven by the lack of answers to some fundamental questions, such as understanding the unification of the forces of nature or the mechanism of baryogenesis, as well as by hints of new physics appearing in anomalous experimental observations. The latter become especially intriguing when the known Standard Model (SM) background (BG) can be reduced to negligible levels so that even the observation of a few events can be attributed to BSM effects.

The flavor-changing rare decays of kaons are exceptionally promising examples of such discovery channels given their highly suppressed SM rates. In particular, the KOTO Collaboration has recently reported a possible observation of three anomalous events in the search for $K_{L} \rightarrow \pi^{0} \nu \bar{\nu}$ [1], in which no SM signal was expected at the current level of the experimental sensitivity. If taken as a

\footnotetext{
felixk@slac.stanford.edu

's.trojanowski@sheffield.ac.uk
}

Published by the American Physical Society under the terms of the Creative Commons Attribution 4.0 International license. Further distribution of this work must maintain attribution to the author(s) and the published article's title, journal citation, and DOI. Funded by SCOAP. signal, this observation would indicate that the relevant branching fraction exceeds the SM prediction by about 2 orders of magnitude [2]. It has been quickly noticed that the discrepancy could be resolved by introducing a new light long-lived particle (LLP) with mass of order $100 \mathrm{MeV}$, light enough to be produced in kaon decays. By properly adjusting the lifetime of the LLP, one can explain the neutral kaon anomaly while avoiding stringent bounds on such models from searches for charged kaon decays.

In this study, we illustrate how such proposed solutions to the KOTO anomaly could be thoroughly probed by the recently approved ForwArd Search ExpeRiment, or FASER [3-5]. The FASER experiment will search for LLPs abundantly produced in the far-forward direction of the Large Hadron Collider (LHC) during its upcoming run 3. Several features of its setup make it an ideal probe for light LLPs produced in kaon decays: (i) Given a large expected boost factor of light particles traveling towards FASER, and the location of the detector, the experiment's sensitivity is optimal for the precise combination of the lifetime and mass that are required to fit the anomaly. In particular, for high-energy LLPs with $E \sim 1 \mathrm{TeV}$ that are produced at the ATLAS IP, the sensitivity reach of FASER is maximized for

$$
\frac{\tau_{\mathrm{LLP}}}{m_{\mathrm{LLP}}} \sim \frac{0.1 \mathrm{~ns}}{100 \mathrm{MeV}} \text { (best FASER reach). }
$$


(ii) The FASER sensitivity additionally benefits from the $\mathcal{O}(150 \mathrm{~m})$ long part of the LHC beam pipe, in which forward-going neutral kaons can travel and decay before being absorbed, as well as the presence of additional LLP production modes at the LHC. (iii) The FASER detector has the capability to not only detect LLP decays into charged particles but also into photon pairs, due to its dedicated preshower detector. As a result, during run 3 of the LHC, FASER can turn the three currently observed anomalous events at KOTO into up to $\mathcal{O}\left(10^{4}\right)$ spectacular high-energy LLP decay events with expected negligible BG.

The rest of this paper is organized as follows. In Secs. II and III we provide more details about the KOTO anomaly and the FASER experiment, respectively. The FASER sensitivity reach in several selected BSM scenarios is presented in Sec. IV. In Sec. V, we further illustrate the possible interplay between FASER and searches for LLPs in kaon factories. We conclude in Sec. VI. Several useful expressions for BSM meson decay branching fractions are listed in the Appendix.

\section{KOTO EXPERIMENT AND THE ANOMALY}

The KOTO experiment [6] has been designed to study decays of kaons into neutral pions and a neutrinoantineutrino pair, $K_{L} \rightarrow \pi^{0} \nu \bar{\nu}$. The kaons are produced in collisions of $30 \mathrm{GeV}$ protons from the Japan Proton Accelerator Research Complex [7] (J-PARC) main ring accelerator with the target made out of gold. A highintensity beam of neutral kaons produced at an angle of $16^{\circ}$ with respect to the proton beam line is created with the use of dedicated collimators. At a distance of about $24 \mathrm{~m}$ away from the target, the $\sim 3 \mathrm{~m}$ long vacuum chamber of the KOTO detector begins. Here, kaon decays are identified by detecting photons produced in prompt neutral pion decays.

The data collected by the KOTO Collaboration in 2015 allowed them to obtain the leading bound on the branching fraction of the aforementioned decay process. At the 90\% confidence level (CL) it reads [8]

$$
\mathcal{B}_{\text {KOTO,bound }}\left(K_{L} \rightarrow \pi^{0} \nu \bar{\nu}\right) \leq 3 \times 10^{-9} \text {. }
$$

Notably, Eq. (2) is consistent with the SM prediction which remains about 2 orders of magnitude lower, $\mathcal{B}_{\mathrm{SM}}\left(K_{L} \rightarrow \pi^{0} \nu \bar{\nu}\right)=(3.4 \pm 0.6) \times 10^{-11} \quad$ [9]. A similar bound on the two-body decay $K^{0} \rightarrow \pi^{0} X$ has also been obtained, where $X$ is a stable or long-lived BSM bosonic particle,

$$
\mathcal{B}_{\text {KOTO,bound }}\left(K_{L} \rightarrow \pi^{0} X\right) \lesssim 2.4 \times 10^{-9} \text {. }
$$

In the subsequent analysis of the data from 2016-2018 [1], however, a total of four candidate events were found, only one of which had the properties of BG. If taken as a signal, the remaining three anomalous events point to the branching fraction which significantly exceeds the SM prediction. At the $95 \% \mathrm{CL}$ it is given by [2]

$$
\mathcal{B}_{\text {KOTO,anom. }}\left(K_{L} \rightarrow \pi^{0} \nu \bar{\nu}\right)=\left(2.1_{-1.7}^{+4.1}\right) \times 10^{-9} .
$$

Importantly, in the SM the aforementioned neutral kaon decay mode is also related by the isospin symmetry to the value of the branching fraction of a charged kaon decay into a pion $\pi^{+}$and a neutrino-antineutrino pair, which proceeds via the same parton level process $s \rightarrow d \nu \bar{\nu}$. The relevant so-called Grossman-Nir (GN) bound [10] reads

$$
\mathcal{B}\left(K_{L} \rightarrow \pi^{0} \nu \bar{\nu}\right)<4.3 \mathcal{B}\left(K^{+} \rightarrow \pi^{+} \nu \bar{\nu}\right) .
$$

The current upper limit on the $K^{+}$decay branching fraction is obtained from the results of the E949 experiment, which observed several charged kaon decay events $[11,12]$, and it is given by $\mathcal{B}_{\text {E949, bound }}\left(K^{+} \rightarrow \pi^{+} \nu \bar{\nu}\right)<3.35 \times 10^{-10}$ at 90\% CL. This is consistent with the SM expectation $\mathcal{B}_{\mathrm{SM}}\left(K^{+} \rightarrow \pi^{+} \nu \bar{\nu}\right)=(8.4 \pm 1) \times 10^{-11}$ [9]. An improved analysis has also recently been preliminarily presented by the NA62 Collaboration with the corresponding branching fraction measured to be at the $68 \%$ CL [13],

$$
\mathcal{B}_{\mathrm{NA} 62}\left(K^{+} \rightarrow \pi^{+} \nu \bar{\nu}\right)=0.47_{-0.47}^{+0.72} \times 10^{-10},
$$

and the upper bound at the 95\% CL given by $\mathcal{B}_{\mathrm{NA} 62 \text {,bound }}\left(K^{+} \rightarrow \pi^{+} \nu \bar{\nu}\right)<2.44 \times 10^{-10}$. The measurements of the charged kaon decay branching fraction, together with the GN bound Eq. (5), lead to (2-3) $\sigma$ tension with the anomalous observation by the KOTO Collaboration, cf. Eq. (4), depending on how the possible impact of new physics is taken into account [2]. A number of BSM scenarios have been proposed to explain this discrepancy [2,14-31].

Among these scenarios, a prominent role is played by models predicting the existence of a new LLP with the mass $m_{X} \sim 100 \mathrm{MeV}$, which can be produced in rare kaon decays, $K_{L} \rightarrow \pi^{0} X$ [2]. In particular, if such a light BSM particle has a lifetime of order $\tau_{X} \sim 0.1 \mathrm{~ns}$, it can be effectively stable and invisible in the search performed by the KOTO Collaboration. At the same time, $X$ will typically decay inside the E949 and NA62 detectors, and it does not contribute to the measured branching fraction of $K_{L}^{+} \rightarrow \pi^{+} \nu \bar{\nu}$. This leads to an apparent violation of the GN bound when the results of these experiments are compared with each other, which is further referred to as the lifetime solution to the anomaly.

A very long-lived $X$ can also explain the anomaly while avoiding the bounds from past beam-dump searches. In addition, if its mass is close to the pion mass, it can escape detection in E949 and NA62 experiments by hiding in the SM BG [32]. Another distinct possibility is to allow $K_{L}$ 
decays into neutral dark states, $K_{L} \rightarrow X_{1} X_{2}$. Subsequent $X_{i}$ decays that produce photons can be detected in the KOTO electromagnetic calorimeter and resemble a neutral pion signature [26]. In this case, charged kaon three-body decays can be suppressed or even kinematically forbidden.

Below, we analyze the prospect of probing such selected BSM scenarios in the FASER experiment at the LHC.

\section{FASER AND FASER 2 EXPERIMENTS}

The FASER experiment [3-5] has been proposed to search for LLPs $[33,34]$ produced in the forward region of the LHC [35-41], as well as to study interactions of highenergy neutrinos [42-44]. It utilizes the fact that light and high-energy particles produced in $p p$ collisions at the LHC, and e.g., subsequent decays of light mesons $M$, will typically travel in the forward direction, as dictated by their estimated angular spread around the beam collision axis, $\theta_{X} \sim m_{M} / E_{X} \ll 1$. As a result, even a small detector placed along the beam collision axis can search for displaced decays of LLPs, provided that it is shielded to suppress the SM BG. The FASER detector will operate during the LHC run 3 in the former service tunnel TI12 located at a distance $L=480 \mathrm{~m}$ away from the ATLAS IP. Below, we also show the sensitivity reach for the same detector with continued operation during the HighLuminosity LHC phase (HL-LHC). We refer to this version of the experiment as FASER HL. We also present the results obtained for a possible larger version of the detector to operate during HL-LHC, dubbed FASER 2. In particular, we assume a cylindrical decay volume with length $\Delta$, radius $R$, and an integrated luminosity $\mathcal{L}$ for each of the three aforementioned cases:

FASER : $\Delta=1.5 \mathrm{~m}, \quad R=10 \mathrm{~cm}, \quad \mathcal{L}=150 \mathrm{fb}^{-1}$, FASER HL : $\Delta=1.5 \mathrm{~m}, \quad R=10 \mathrm{~cm}, \quad \mathcal{L}=3 \mathrm{ab}^{-1}$,

FASE R 2: $\Delta=5 \mathrm{~m}, \quad R=1 \mathrm{~m}, \quad \mathcal{L}=3 \mathrm{ab}^{-1}$.

Our main focus in this study is to highlight possible complementarity between FASER searches for LLPs and kaon factories, with a particular emphasis on the recently observed KOTO anomaly. It is then useful to briefly summarize the main advantages of FASER that make it an ideal tool to study related BSM scenarios predicting a new unstable light species:

(i) FASER has the capability to study diphoton final states in LLP decays, on top of the more-oftenconsidered electron-positron pairs.

(ii) Forward-going kaons produced in $p p$ collisions at the ATLAS IP can travel about $140 \mathrm{~m}$, and hence decay with sizable probability, before they are absorbed. Additional production modes can further improve detection prospects. (iii) FASER's sensitivity is optimal for typical LLP mass and lifetime proposed to explain the KOTO anomaly, cf. discussion in Sec. II.

The signatures that we focus on consist of LLP $X$ decays into mainly $\gamma \gamma$ or $e^{+} e^{-}$pairs that can be resolved in the detector. For sufficiently large energy, which we take to be $E_{X} \gtrsim 100 \mathrm{GeV}$, the search at the FASER location can be considered BG-free $[4,5]$. In the following, we assume a $100 \%$ detection efficiency. Notably, the preliminary efficiency studies show that it typically has a minor impact on the sensitivity reach plots [4].

\section{A. FASER capabilities to study the $\gamma \gamma$ final state}

As far as the $\gamma \gamma$ final state is concerned, a very good separation efficiency between the photons is achieved thanks to a dedicated preshower detector placed in front of the FASER calorimeter, i.e., $2 \mathrm{~m}$ after the end of the FASER decay vessel. A preliminary relevant discussion can be found in Ref. [37] in the context of a FASER search for axionlike particles with the dominant diphoton coupling. As shown there, taking into account a finite separation resolution between the two $\gamma \mathrm{s}$ of order $\delta \sim 200 \mathrm{~nm}$, which is achievable in FASER, has a negligible impact on the sensitivity reach. Instead, a substantial effect was expected only if the resolution was worse than about $1 \mathrm{~mm}$.

Additionally, LLP decays into photons could also be proved in the single-photon channel. Importantly, single high-energy BG photons with $E_{\gamma} \gtrsim 100 \mathrm{GeV}$ at a FASER location are typically associated with time-coincident muons activating veto layers. Hence, a large number of very energetic photon pairs produced in LLP decays even closer to the preshower detector, which could be misidentified as a single EM shower, would still be indicative of new physics.

\section{B. Forward-going kaons at the LHC}

It is also important to stress that forward-going kaons produced at the ATLAS IP will not be immediately absorbed. Instead, they can travel a long distance through the LHC beam pipe, which allows them to decay with a sizable probability. In particular, for neutral kaons produced within the angular coverage of FASER, $\theta_{K}<$ $\theta_{\text {FASER }} \approx 0.2 \mathrm{mrad}$, the closest element of the LHC infrastructure that they hit is the TAN neutral particle absorber placed at a distance $140 \mathrm{~m}$ away from the ATLAS IP. We take this into account in our modeling along with the TAS absorber placed $20 \mathrm{~m}$ away from the IP that affects neutral kaons produced with larger $\theta_{K}$. The TAS also marks the end of the region where charged kaons are not deflected away by strong LHC magnets.

Below, we also illustrate how in specific models additional production modes of LLPs, for example, in heavy meson decay, can further improve FASER sensitivity. 


\section{Lifetime regime}

Last but not least, it is worth highlighting that in order for the LLP with $m_{X} \sim 100 \mathrm{MeV}$ and energy $E_{X} \sim \mathrm{TeV}$ to reach FASER, the required lifetime is of order $\tau_{X} \sim L /\left(\gamma_{X} c\right) \sim 0.1 \mathrm{~ns}$. This precisely corresponds to the sweet spot between the KOTO and NA62 searches discussed in Sec. II. Therefore, as we will see below, it is not a surprise that FASER can effectively exclude many such explanations of the anomaly even with the first $10 \mathrm{fb}^{-1}$ of data. Instead, in the case of discovery, FASER can confirm the anomaly with large statistics of related LLP decay events. This can reach up to $\mathcal{O}\left(10^{4}\right)$ events in FASER during LHC run 3, while it could grow even larger for FASER HL and FASER 2.

\section{FASER SENSITIVITY REACH}

As discussed in Sec. II, if the anomalous neutral kaon decay events observed in the KOTO experiment are confirmed, this would require a BSM explanation. In the following, we illustrate how such models can be independently probed in FASER. To this end, we focus on several scenarios predicting LLPs.

\section{A. Generic LLP and the lifetime solution}

The simplest BSM scenario proposed to explain the anomaly and to avoid stringent bounds on rare decays of charged kaons employs a new unstable $X$ particle produced in kaon decays, $K_{L} \rightarrow \pi^{0} X$ and $K^{+} \rightarrow \pi^{+} X$. In particular, if the lifetime of $X$ and its mass are of order $\tau_{X} \sim 0.1 \mathrm{~ns}$ and $m_{X} \sim 100 \mathrm{MeV}$, respectively, then $X$ typically decays within the volume of the E949 and NA62 detectors and does not contribute to the measured value of $\mathcal{B}\left(K^{+} \rightarrow \pi^{+} \nu \bar{\nu}\right)$. On the other hand, the probability for $X$ to escape the KOTO decay volume without decaying remains larger [2].

In the left panel of Fig. 1, we show the FASER and FASER HL sensitivity reach contours in a generic scenario, in which $X$ is produced only in kaon decays. We assume that the real branching fraction of a two-body neutral kaon decay is of order $\mathcal{B}\left(K^{0} \rightarrow \pi^{0} X\right) \sim 10^{-8}-10^{-6}$. Its precise value is chosen depending on $m_{X}$ and $\tau_{X}$, as well as the KOTO detector efficiency [8], as detailed in Refs. [2,18]. This is done by requiring that the measured branching fraction fits the KOTO anomaly, cf. Eq. (4), after correcting for finite escape probability from the detector. In the plot, it is also assumed that the charged kaon decay branching fraction saturates the GN bound, cf. Eq. (5). The grayshaded region is excluded by the constraints from the aforementioned searches for $K^{+} \rightarrow \pi^{+} \nu \bar{\nu}$ at E949 [12] and NA62 [13], the untagged $K_{L} \rightarrow \pi^{0} X$ branching fraction [45], beam-dump searches at CHARM [46] and NuCal $[47,48]$ experiments that we implement following Refs. [15,18], and the measurement of the branching
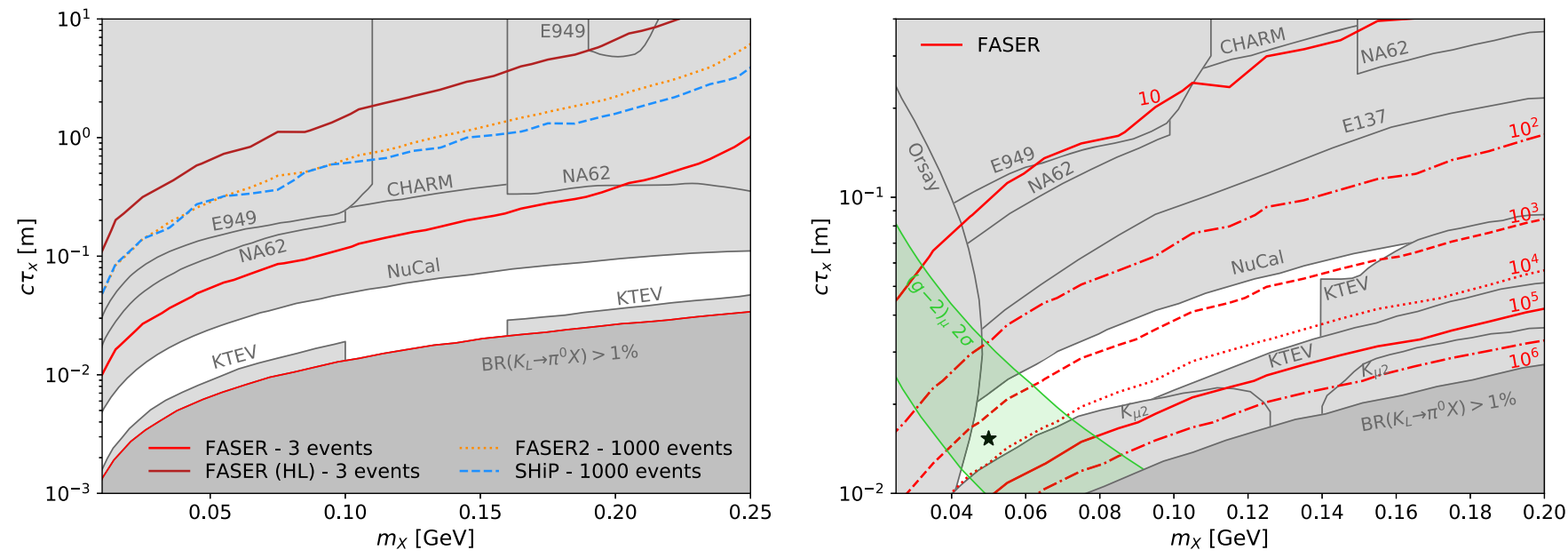

FIG. 1. Results for the lifetime solution to the KOTO anomaly (white regions in the plots) presented in the $\left(m_{X}, c \tau_{X}\right)$ plane, where $m_{X}$ and $\tau_{X}$ correspond to the LLP mass and lifetime, respectively. The current bounds are shown as gray-shaded regions. Left panel: Reach for the generic $X$ scenario in which a new light and unstable particle is produced specifically in the $K_{L}$ and $K^{+}$decays, cf. discussion in Sec. IV A. From top to bottom, dark and light red solid lines correspond to the upper limit in $c \tau_{X}$ of the sensitivity reach of the FASER HL and FASER experiments, respectively. The experiments can probe scenarios relevant for the entire white region in the plot below the sensitivity lines. Also shown are the lines with a fixed number of events $N_{\mathrm{ev}}=1000$ expected for the FASER 2 (orange dotted line) and SHiP (blue dashed line) experiments. The number of events in both cases grow for lower values of $c \tau_{X}$ in the white region of the plot. At the bottom of the plot, the dark red solid line marks the region of the parameter space which is excluded by the untagged $K_{L} \rightarrow \pi^{0} X$ branching fraction. Right panel: Event contours [from top to bottom: 10 (solid), $10^{2}$ (dashdotted), $10^{3}$ (dashed), $10^{4}$ (dotted), $10^{5}$ (solid), and $10^{6}$ (dash-dotted)] at FASER at the LHC run 3 for the dark Higgs boson model with nonuniversal couplings to the SM leptons and quarks, as discussed in Sec. IV B. The green-shaded band corresponds to the $2 \sigma$ fit to the $(g-2)_{\mu}$ anomaly. 
TABLE I. The number of $X$ decay events in FASER (FASER 2) and the integrated luminosity of $\mathcal{L}=150 \mathrm{fb}^{-1}\left(\mathcal{L}=3 \mathrm{ab}^{-1}\right)$ for various production modes of the LLP. The number of events for FASER HL are 20 times larger than for FASER during LHC run 3 . The results correspond to the model discussed in Sec. IV B and to the benchmark values of the model parameters given in the text and indicated in the table. The scenario can explain both the KOTO and $(g-2)_{\mu}$ anomalies.

\begin{tabular}{|c|c|c|c|c|c|c|c|}
\hline \multirow[b]{2}{*}{ Experiment } & \multicolumn{2}{|c|}{ Benchmark } & \multicolumn{4}{|c|}{$X$ prod. in meson decays and decay in FASER } & \multirow{2}{*}{$\frac{\text { Proton brem }}{p p \rightarrow X+\cdot}$} \\
\hline & $m_{X}[\mathrm{MeV}]$ & $c \tau_{X}[\mathrm{~cm}]$ & $K_{L} \rightarrow \pi^{0} X$ & $K^{+} \rightarrow \pi^{+} X$ & $\eta, \eta^{\prime}, K_{S} \rightarrow \pi^{0} X$ & $b \rightarrow s X$ & \\
\hline FASER & 50 & 15 & 150 & 50 & 650 & 2000 & 135 \\
\hline FASER 2 & 50 & 1.5 & $80 \mathrm{k}$ & $30 \mathrm{k}$ & $250 \mathrm{k}$ & $11 \mathrm{M}$ & $16 \mathrm{k}$ \\
\hline
\end{tabular}

fraction for $K_{L} \rightarrow \pi^{0} \gamma \gamma$ by KTEV [49], assuming that $X$ decays dominantly into a $\gamma \gamma$ final state. ${ }^{1}$

The anomalous KOTO events can be fitted within the remaining portion of the parameter space shown in Fig. 1. Notably, the reconstructed transverse momentum of the pion can more easily resemble the observed distribution by KOTO when $m_{X} \lesssim 180 \mathrm{MeV}$ [2], or even $m_{X} \lesssim m_{\pi}$ [23].

In order to obtain the FASER sensitivity reach, we generate the meson spectra using EPOS-LHC [50] as implemented in the simulation package CRMC [51] and subsequently decay the mesons using the branching fractions obtained as discussed above. As can be seen, the FASER experiment operating during the LHC run 3 can already cover the entire region of the parameter space corresponding to the anomaly. The relevant expected number of events can be as large as $\mathcal{O}\left(10^{2}\right)$ for FASER and grow by an additional factor of 20 and a few hundred for FASER HL and FASER 2, respectively. Notably, thanks to the use of the dedicated preshower detector, FASER can probe this scenario even for leptophobic $X$, which decays dominantly into a diphoton pair, as assumed in the bounds shown in the left panel of Fig. 1.

The lifetime solution to the KOTO anomaly can also be explored by other experiments dedicated to LLP searches, although this can be limited by the lack of diphoton signal detection capabilities. We illustrate this in the left panel of Fig. 1 for the proposed SHiP experiment [52] to operate in a similar time frame to FASER HL and FASER 2. The sensitivity of SHiP is analyzed by conservatively requiring that the kaons decay within one nuclear interaction length in the SHiP target made out of molybdenum, $\lambda=15.25 \mathrm{~cm}$. We show the relevant contour line corresponding to a fixed number of expected events, $N_{\mathrm{ev}}=1000$, similarly to the FASER 2 line also shown in the plot. The number of events grows larger for lower values of the LLP lifetime within the allowed region in the $\left(m_{X}, c \tau_{X}\right)$ plane. Interestingly, although kaon absorption in

\footnotetext{
${ }^{1}$ In particular, the NuCal bounds shown in Fig. 1 have been obtained following a "conservative" approach discussed in Ref. [15] and by taking into account $X$ production in decays of kaons (left panel) or all the relevant mesons (right panel). We note that the bounds from hadronic beam dumps are subject to uncertainties in modeling of the high-energy tail of the meson spectrum in the target.
}

the target at distances greater than $\lambda$ could limit SHiP sensitivity, a large number of protons on target (POT), $N_{\text {POT }}=2 \times 10^{20}$, as well as the size of the decay volume, $\Delta \simeq 50 \mathrm{~m}$, sufficiently compensate for this effect. The number of expected events in SHiP could be further enhanced once more detailed modeling of the kaon propagation in the target is performed.

\section{B. Specific example employing 2HDM}

Once a specific model of the LLP that corresponds to the aforementioned lifetime solution is considered, typically more production and decay modes appear for $X$ that should be taken into account. This has an impact on present constraints on such $X$ but can also significantly increase the expected number of events in the future detectors. We illustrate this below for FASER employing the model with a leptophilic dark scalar discussed in Ref. [18]. Interestingly, such a scalar with $40 \mathrm{MeV} \lesssim m_{X} \lesssim 70 \mathrm{MeV}$ and $\tau_{X} \sim$ $0.1 \mathrm{~ns}$ could simultaneously explain the recently observed KOTO events and the measurement of the anomalous magnetic moment of the muon, $(g-2)_{\mu}$ [53].

The model of our interest can effectively be described by three parameters corresponding to the LLP mass, as well as its coupling constants to leptons, $\epsilon_{\ell}$, and quarks, $\epsilon_{q}$. The relevant Lagrangian reads

$$
\begin{aligned}
\mathcal{L} \supset & -m_{X}^{2} X^{2}+\sum \epsilon_{q} \frac{m_{q}}{v} X \bar{q} q+\sum \epsilon_{\ell} \frac{m_{\ell}}{v} X \bar{\ell} \ell \\
& +\epsilon_{W} \frac{m_{W}^{2}}{v} X W_{\mu}^{+} W^{\mu-}
\end{aligned}
$$

where the SM Higgs boson vev is equal to $v \simeq 246 \mathrm{GeV}$ and we set $\epsilon_{W}=\epsilon_{q}$. The couplings $\epsilon_{\ell}$ and $\epsilon_{q}$ can arise as the mixing angles in the scalar sector of the type- $X$ two Higgs doublet model (2HDM). In particular, the model allows one to disjointly treat interactions of $X$ with the SM leptons and quarks.

Assuming a hierarchy between the couplings, $\epsilon_{\ell} \gg \epsilon_{q}$, allows one to reduce the lifetime of $X$, which is governed by the leptonic coupling $\epsilon_{\ell}$. Thanks to this, stringent bounds from hadronic beam-dump searches can be avoided and the kaon decay branching fraction into $X$ can be kept low, as it depends on $\epsilon_{q} \sim 10^{-3}-10^{-2}$. In particular, for 
$\epsilon_{\ell} \sim \mathcal{O}(1)$ the most important bounds on long-lived $X$ come from electron beam-dump experiments E137 [54] and Orsay [55]. However, they do not exclude scenarios with $\tau_{X} \sim 0.1 \mathrm{~ns}$.

The dominant decay mode for $X$ in the considered mass regime is into the $e^{+} e^{-}$final state, although a loop-induced decay into the $\gamma \gamma$ pair can take values of up to $\mathcal{O}(10 \%)$ of the decay branching fraction of $X$. The additional bounds from the KTEV search for $K_{L} \rightarrow \pi^{0} e^{+} e^{-}$decays exclude a part of the region of the parameter space of the model with $m_{X} \gtrsim 140 \mathrm{MeV}$ [56], although they are not relevant for lighter $X$. We employ the decay width of $X$ following Ref. [18].

A relative smallness of $\epsilon_{q}$ is consistent with only a few events currently observed in KOTO. However, as far as FASER is concerned, additional production modes of $X$ can become more important than rare kaon decays, therefore increasing the expected number of signal events. In particular, when obtaining the FASER sensitivity reach, we take into account the following production channels:

\section{Meson decays}

In our modeling, we include rare two-body decays of charged and neutral long-lived kaons that propagate in the LHC beam pipe, as discussed in Sec. III. We also analyze the impact of short-lived kaon decays, $K_{S} \rightarrow \pi^{0} X$. Notably, the relevant BSM branching fraction of $K_{S}$ is suppressed with respect to $K_{L}$ by an additional factor of order $\mathcal{O}\left(10^{-4}\right)$ due to a larger value of the total decay width of $K_{S}$. On the other hand, short-lived kaons can more easily decay prior to hitting any element of the LHC forward infrastructure.

On top of kaons, a number of other mesons can be abundantly produced in the forward direction of the LHC. In particular, we study prompt rare two-body decays $\eta \rightarrow \pi^{0} X$ and $\eta^{\prime} \rightarrow \eta X$, as well as inclusive decays of $B$ mesons into final states containing strange hadronic states, $b \rightarrow s X$, where the light meson spectra were obtained using EPOS-LHC, while the $b$-quark spectrum was obtained using PYTHIA8 [57,58]. We implement the kaon and $B$-meson branching fractions following Ref. [35], while for $\eta$ mesons we employ the results from Ref. [15]. We give the relevant expressions for the branching fractions in the Appendix.

The dominant contribution to the $K$ and $B$ decay widths into $X$ corresponds to a top- $W$ loop. Unlike for kaon decays, the relevant branching fractions of $B$ mesons do not suffer from a strong CKM suppression, $V_{t b}^{2} \gg V_{t d}^{2}$, which makes this channel the dominant production mode despite the suppressed $b$-quark production rate and the broader $p_{T}$ spectrum. Instead, the decays of $\eta$ mesons are suppressed for small Yukawa-like couplings of $X$ to the first-generation quarks and for a loop-induced coupling to gluons. Similarly to $K_{S}$, however, prompt decays of $\eta$ mesons make them a non-negligible production mode of $X$.

\section{Scalar bremsstrahlung}

Another production mode of light and high-energy scalar BSM particles produced in the forward direction of the LHC is through their bremsstrahlung in proton-proton collisions. We study this process following the discussion in Ref. [59] and find that the relevant contribution to the event rate in FASER is typically of order a few $\%$ or smaller.

\section{Lepton-induced production}

We have also analyzed a number of production modes that depend on the lepton coupling, $\epsilon_{\ell} \sim \mathcal{O}(1)$. These include loop-induced Primakoff production from highenergy photons hitting the TAN, cf. Ref. [37], as well as various secondary processes employing electrons and muons pair-produced in the absorber material or traversing the rock shielding of FASER. However, since the difference in $\epsilon_{\ell}$ and $\epsilon_{q}$ is typically not sufficient to compensate for a large Yukawa suppression of the corresponding coupling constants, as well as due to additional suppression factors relevant for these production modes, we have found that they play a subdominant role for high-energy scalars of our interest.

In the right panel of Fig. 1, we show the contours with the different number of events in FASER, $N=10,10^{2} \ldots$, that correspond to the total integrated luminosity of $\mathcal{L}=$ $150 \mathrm{fb}^{-1}$ for the LHC run 3. In the white region of the plot, the KOTO anomaly can be explained without violating current bounds shown as the gray-shaded region, following Ref. [18]. In addition, we have added the constraint from the NuCal experiment following Ref. [15]. The greenshaded band in the plot indicates the region in the parameter space of the model in which the $(g-2)_{\mu}$ anomaly can be resolved. As can be seen, FASER can detect up to $\mathcal{O}\left(10^{4}\right)$ LLP decays in the region of the parameter space corresponding to both the anomalies. In this case, even a few weeks of the operation of the experiment would be enough to test such scenarios. Once more data are accumulated, the entire allowed region of the parameter space will be covered with at least a few hundred expected events.

The breakdown of the number of expected events in FASER and FASER 2 for different production modes of $X$ is shown in Table I for the benchmark scenario with $m_{X}=50 \mathrm{MeV}, \epsilon_{q}=0.016$, and $\epsilon_{\ell}=1.22$. The values of the parameters of the model have been chosen so that both the KOTO and $(g-2)_{\mu}$ anomalies can be fitted to their central values [18]. The dominant production mode in FASER, in this case, is due to rare $B$ meson decays, although decays of lighter mesons give a contribution of order $30 \%$ in total. This is dominated by rare decays of $\eta$ mesons. Instead, the kaon decays give only a few \% contribution. 


\section{A very long-lived dark scalar}

If a new LLP is produced in rare kaon decays with the mass close to the pion mass, $m_{X} \sim m_{\pi}$, and the relevant branching fraction fitting the anomaly, cf. Eq. (4), the stringent NA62 and E949 bounds on $K^{+}$decays can be avoided even for a very long-lived or effectively stable $X$. This is due to increased BG in the respective searches for $\mathrm{K}^{+}$decays (see the discussion in Ref. [2] and references therein). In addition, such scenarios can also escape constraints from beam-dump searches provided that $\tau_{X}$ is large enough, and the LLPs typically overshoot the detector.

An interesting example of such a scenario that can fit the KOTO anomaly is a dark Higgs boson $X$ with a universal mixing angle with the SM species, cf. Eq. (7) with $\epsilon_{\ell}=\epsilon_{q}=\epsilon_{W}$ and Ref. [15] for further discussion. In this scenario, the lifetime of the LLP is typically large, $c \tau_{X} \sim 100 \mathrm{~km}$. As a result, probing this model goes beyond the capabilities of FASER and FASER 2 experiments, which are designed to focus on more short-lived BSM species.

On the other hand, in the long-lifetime regime relevant here, the sensitivity reach in searches for displaced decays of $X$ can be improved by increasing the size of the detector and its angular coverage. We illustrate this in the left panel of Fig. 2, where we show the expected reach of proposed future Codex-b [60], MATHUSLA [61], and SHiP [52] experiments in their searches for dark Higgs boson decays into electron-positron pairs, $X \rightarrow e^{+} e^{-}$, following Ref. [33].

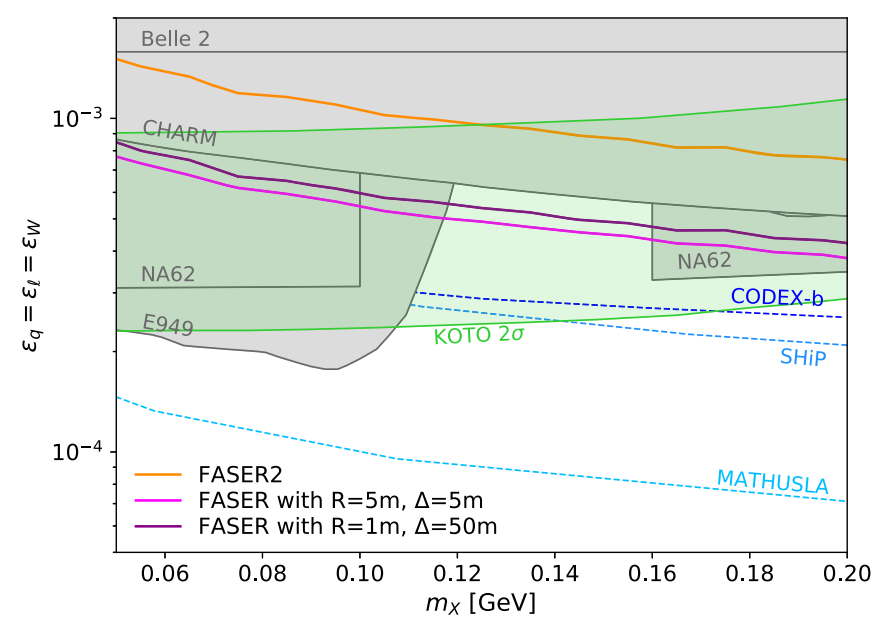

For comparison, we also present the expected sensitivity of the enlarged FASER 2 detector assuming the total integrated luminosity of $\mathcal{L}=3 \mathrm{ab}^{-1}$ relevant for HLLHC. As can be seen, probing the region in the parameter space of the model that corresponds to the KOTO anomaly would require a quite substantial increase in the detector radius, $R \gtrsim 5 \mathrm{~m}$, or its length, $\Delta \gtrsim 50 \mathrm{~m}$, with respect to the design discussed in Sec. III. On the other hand, for lower values of $\tau_{X}$, even a much smaller and properly placed detector can have very good detection prospects, as we show for other scenarios discussed in this study.

\section{D. $K_{L}$ decays into dark sector particles}

A different approach to the BSM explanation of the KOTO anomaly has been proposed in Refs. [25,26]; this approach employs two-body decays of neutral kaons into dark species, $K_{L} \rightarrow \psi_{1} \psi_{2}$. The relevant three-body decay processes of charged kaons, e.g., $K^{+} \rightarrow \pi^{+} \psi_{1} \psi_{2}$, can then be kinematically suppressed or even forbidden depending on the masses of the $\psi_{i}$ s. The anomaly can be fitted if at least one of the dark species is unstable and can mimic the diphoton decay signature of neutral pions inside the KOTO detector.

In the following, we focus on the model in which a nondiagonal coupling of kaons to two dark fermionic states $\psi_{i}$ arises due to a vector portal and a new gauge field $X_{\mu}$ that mixes with the SM $Z$ boson [62-64],

$$
\mathcal{L} \supset \frac{1}{2} m_{X}^{2} X_{\mu} X^{\mu}-\epsilon_{Z} m_{Z}^{2} X_{\mu} Z^{\mu}
$$

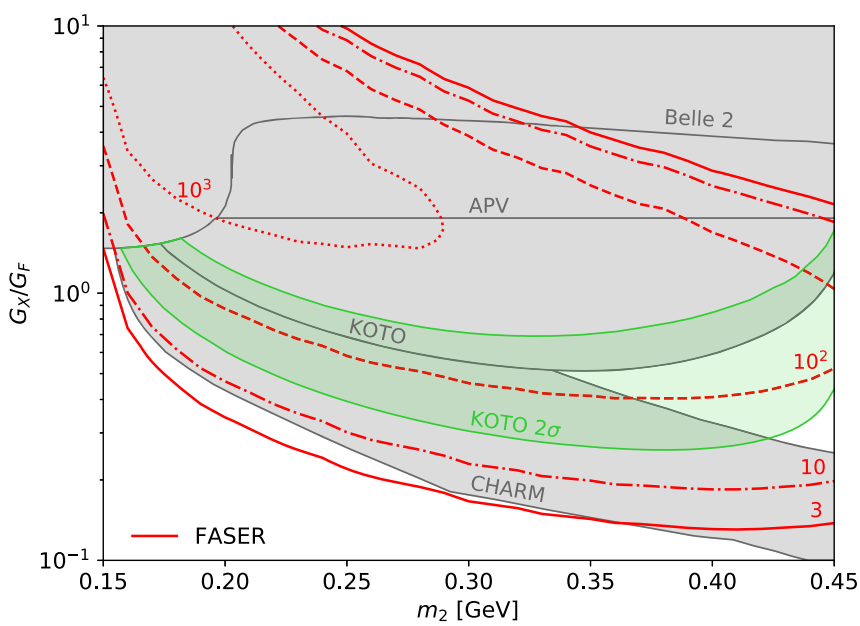

FIG. 2. Results for the models discussed in Secs. IV C and IV D. In both panels, the $2 \sigma$ regions of the parameter space in which the KOTO anomaly can be explained are shown as the green-shaded bands. Left panel: Reach for the dark Higgs boson model discussed in Sec. IV C. The sensitivity contour for FASER 2 is shown with the orange solid line, while purple and yellow solid lines correspond to larger versions on FASER 2 with the radius $R=5 \mathrm{~m}$ or length $\Delta=50 \mathrm{~m}$, respectively. The current bounds are shown as gray-shaded regions following Ref. [15]. The blue dashed lines correspond to the expected sensitivity of other proposed future experiments (from top to bottom): CODEX-b, SHiP, and MATHUSLA. Right panel: Event contours [from outside to inside: 3 (solid red line), 10 (dash-dotted), 100 (dashed), 1000 (dotted)] at FASER at LHC run 3 for the model with pure dark sector decays of kaons, $K_{L} \rightarrow X_{1} X_{2}$, discussed in Sec. IV D. The current bounds are shown as gray-shaded regions following Ref. [26]. 
where $\epsilon_{Z} \sim 10^{-3}-10^{-2}$ denotes the relevant mixing parameter. After the electroweak symmetry breaking, a new $Z^{\prime}$ gauge boson acquires a mass $m_{Z^{\prime}}^{2}=m_{X}^{2}-\epsilon_{Z}^{2} m_{Z}^{2}$. The interactions of $Z^{\prime}$ with the dark fermions can then be described by

$$
\mathcal{L} \supset g_{X} Z_{\mu}^{\prime}\left(c_{V} \bar{\psi}_{2} \gamma^{\mu} \psi_{1}+c_{A} \bar{\psi}_{2} \gamma^{5} \gamma^{\mu} \psi_{1}\right)+\text { H.c. }
$$

In particular, an effective operator which couples $\psi_{i} \mathrm{~s}$ to $s$ and $d$ quarks is induced at a loop level with $W$ boson exchange [64]. In the following, we set $g_{X}=1$, $c_{V}=0, c_{A}=1, m_{Z^{\prime}}=10 \mathrm{GeV}$, and $m_{2}=11 m_{1}$, where $m_{i} \equiv m_{\psi_{i}}$.

The $\psi_{2}$ decay width and branching fractions for $\psi_{1} \psi_{2^{-}}$ pair production in various meson decays can be found in Ref. [26]. In particular, as discussed therein, the assumption about a relatively large mass splitting between the dark species, $m_{1} \ll m_{2}-m_{\pi^{0}}$, allows one to obtain about an $80 \%$ branching fraction of two-body decays $\psi_{2} \rightarrow \psi_{1} \pi^{0}$. The subsequent prompt decays of high-energy neutral pions with $E_{\pi^{0}} \gtrsim 100 \mathrm{GeV}$ generate a diphoton signature inside the FASER detector.

In the right panel of Fig. 2, we show the contours with the expected number of events in FASER in the parameter space spanned by $m_{2}$ and $G_{X} / G_{F}$ parameters, where $G_{F}$ is the Fermi coupling constant and $G_{X}=$ $\left(\epsilon_{Z} g g_{X}\right) /\left(2 \sqrt{2} c_{W} m_{Z^{\prime}}^{2}\right)$ with $g$ corresponding to the weak coupling constant. The region of the parameter space in which a $2 \sigma$ fit can be obtained to the KOTO anomaly is indicated by a green-shaded band in the plot. The current bounds on the model from the CHARM experiment, the previous KOTO search for $K_{L} \rightarrow \pi^{0} \nu \bar{\nu}$, cf. Eq. (2), atomic parity violation (APV) [64], and the Belle-II search for $B \rightarrow K \nu \bar{\nu}$ [65] are shown as the gray-shaded region following Ref. [26]. ${ }^{2}$

The region of the parameter space of the model in which the KOTO anomaly can be explained by rare decays $K_{L} \rightarrow \psi_{1} \psi_{2}$ corresponds to $350 \mathrm{MeV} \lesssim m_{2} \lesssim 450 \mathrm{MeV}$. Here, one requires that $\psi_{2}$ decays within the KOTO detector volume so that it can mimic neutral pion decays. However, such decays that are too fast lead to larger production rates that are already excluded by previous KOTO studies. As a result, the anomalous events can be best fitted for $\tau_{\psi_{2}} \sim(0.01-0.1) \mathrm{ns}$, and such scenarios can be well tested in FASER. In particular, as shown in Fig. 2, one expects $\mathcal{O}(100)$ of high-energy visible $\psi_{2}$ decay events in the detector for LHC run 3.

\footnotetext{
${ }^{2}$ As discussed in Ref. [26], a more detailed simulation of the $\mathrm{CHARM}$ and $\mathrm{NuCal}$ experiments is needed to refine the lower part of the gray-shaded region in Fig. 2. This, however, is expected to have a small impact on the upper part of the plot corresponding to the KOTO anomaly.
}

\section{BEYOND THE ANOMALY-ALP COUPLING TO SU $(2)_{W}$ GAUGE BOSONS}

The complementarity between FASER and kaon factories extends beyond possible explanations to the currently observed anomalous events in the KOTO experiment. In particular, more general BSM scenarios leading to twobody kaon decays, $K \rightarrow \pi^{0} X$ and $K^{+} \rightarrow \pi^{+} X$, can be constrained in searches for detector-stable $X$ acting as neutrino impostors, as well as in studies focused on displaced decays $X \rightarrow \gamma \gamma$ leading to $4 \gamma$ or $\pi^{+}+2 \gamma$ signatures observed in the detector. Some examples of such studies have recently been discussed in Ref. [25] for models with axionlike particles (ALPs), $X \equiv a$, which couple dominantly to gluons or $S U(2)_{W}$ gauge bosons. The sensitivity reach of FASER in the former scenario has been studied in Ref. [38].

In the following, we will focus on the latter model in which the ALP couples to the SM field strength tensor $W_{\mu \nu}^{a}$ of the $S U(2)$ group,

$$
\mathcal{L} \supset-\frac{1}{2} m_{a}^{2} a^{2}-\frac{g_{a W W}}{4} a W_{\mu \nu}^{a} \tilde{W}_{\mu \nu}^{a} .
$$

The coupling to $W$ bosons in Eq. (10) gives rise to both kaon decays, $s \rightarrow d a$, and $B$-meson decays, $b \rightarrow s a$, via the usual loop diagrams. We implement the relevant branching fractions following Refs. [25,66,67] as discussed in the Appendix.

After the EWSB, additional couplings of $a$ to $\gamma \gamma, Z \gamma$, and $Z Z$ arise with the relative strength dictated by the weak mixing angle. As a result, the ALP can also be produced through the Primakoff process, $\gamma N \rightarrow a \gamma$, employing the diphoton coupling $g_{a \gamma \gamma}=g_{a W W} s_{W}^{2}$, cf. Ref. [37] for relevant discussion for FASER, providing a subleading contribution to the ALP event rate. In contrast, $a$ production in rare $Z$ decays is more isotropic and can be neglected. For the low-mass ALPs of our interest, the dominant decay mode is into two photons, $a \rightarrow \gamma \gamma$ [68].

We present the FASER, FASER HL, and FASER 2 sensitivity reach lines for this model in Fig. 3. Additional dashed contours in the plot correspond to the expected future sensitivity in searches in KOTO and NA62 for the aforementioned two-body BSM kaon decays, $K^{(+)} \rightarrow$ $\pi^{0(+)} a$. Depending on whether the ALP escapes the detector without decaying or decays inside to two photons, the searches focus on $2 \gamma$ or $4 \gamma$ signatures in KOTO, and a $\pi^{+}+0 \gamma$ or $\pi^{+}+2 \gamma$ signal in NA62. We show these searches following Ref. [25]. The current bounds on the model are taken from Refs. [25,69]. The most important constraints in the region of the parameter space of our interest come from the beam-dump experiment E137 and past searches in kaon factories.

As can be seen, FASER will cover the entire region of the parameter space corresponding to KOTO and NA62 searches for detector-stable $a$. It will also independently 


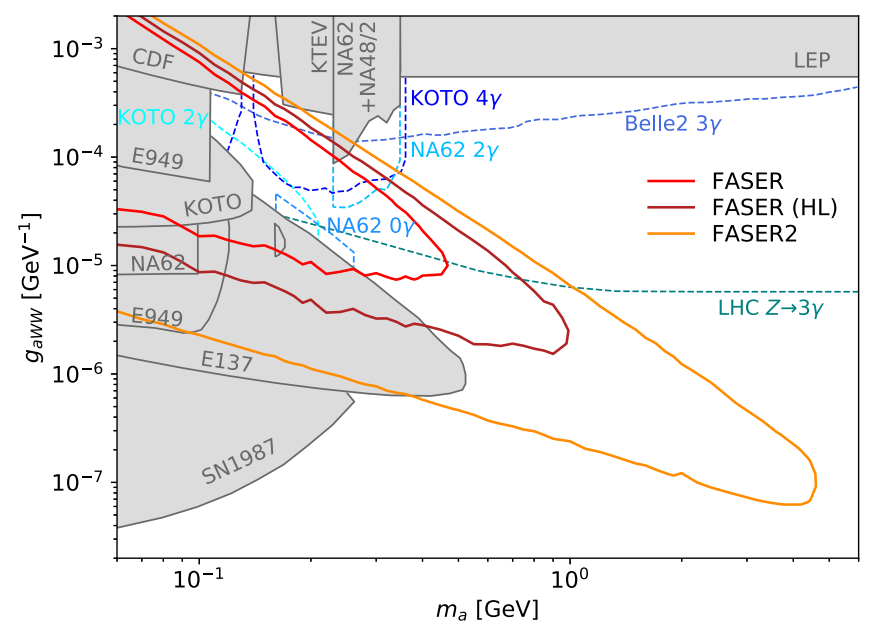

FIG. 3. Sensitivity reach of the FASER (red solid line), FASER HL (brown), and FASER 2 (orange) experiments in the model with axionlike particles coupled to $S U(2)_{W}$ discussed in Sec. V. The currently excluded gray-shaded region and the dashed blue lines corresponding to the future sensitivity reach in the KOTO and NA62 searches for promptly decaying and detector-stable ALPs are taken from Refs. [25,69]. The expected reach of the Belle-II search for the $3 \gamma$ signature is shown following Ref. [69]. The future ATLAS/CMS reach in the $Z \rightarrow 3 \gamma$ signature from production and prompt decays of the ALP to a diphoton pair is shown following Ref. [70].

probe scenarios relevant for unstable ALP decaying inside these detectors. In addition, FASER reach extends towards lower values of the $g_{a W W}$ coupling and larger mass of the ALP. The larger values of the coupling constant will, instead, be probed by e.g., Belle-II searches for the $3 \gamma$ signature from the ALP production and subsequent prompt decay inside the detector, $e^{+} e^{-} \rightarrow \gamma+(a \rightarrow 2 \gamma)$. We show the relevant reach for $50 \mathrm{fb}^{-1}$ in Fig. 3 following Ref. [69]. Similarly, future ATLAS and CMS searches for the $Z \rightarrow \gamma+(a \rightarrow 2 \gamma)$ signature can also cover an important part of the parameter space of the model corresponding to $g_{a W W} \gtrsim 10^{-5} \mathrm{GeV}^{-1}$, as shown in the plot based on Ref. [70].

Both FASER HL and FASER 2 experiments will be able to further improve the detection prospects, as well as constrain scenarios with $m_{a}$ up to $1 \mathrm{GeV}$ or even close to the limit $m_{a} \lesssim m_{B}-m_{K}$, respectively. In a similar time frame, additional bounds on the model can come from e.g., the SHiP experiment (not shown in the plot).

\section{CONCLUSION AND OUTLOOK}

A long-awaited definite experimental discovery of new physics effects should first manifest itself as a clear deviation from the SM predictions and excess over expected BG. In this study, we have focused on recently observed and intriguing anomalous neutral kaon decay events in the KOTO experiment. The current KOTO observation corresponds to a striking excess over the expected SM branching fraction of the decay process $K_{L} \rightarrow \pi^{0} \nu \bar{\nu}$, while it awaits further dedicated analyses to be performed by the KOTO Collaboration and other experiments before it can be fully confirmed. In particular, we have analyzed the prospects of independently probing the relevant BSM scenarios proposed to explain the anomaly in the upcoming FASER experiment at the LHC [3-5].

We have shown that FASER could start probing such models, which predict light and unstable new particles, immediately after the beginning of its operation. The $p p$ collisions at the LHC produce large numbers of kaons that typically hit elements of the infrastructure before decaying unless they go down the beam pipe, i.e., towards FASER. This can lead to a large flux of forward-going LLPs produced in rare kaon decays and in other production modes. The FASER baseline compatible with the predicted lifetime $c \tau_{X}$ of such LLPs, and its excellent diphoton detection capabilities, will then result in up to $\mathcal{O}\left(10^{4}\right)$ highenergy LLP decay events observed during LHC run 3.

Our results are presented for selected distinct BSM scenarios corresponding to the lifetime solution to the KOTO anomaly, as well as for some other models that either predict very long-lived LLPs with a mass close to the pion mass or employ purely dark sector decays of neutral kaons. Interestingly, even in the less promising case of large $\tau_{X}$, FASER can also be indirectly sensitive to KOTOrelated models that predict a larger set of LLPs and simultaneously address a number of outstanding experimental and theoretical issues. An example of such a scenario with below GeV-scale heavy neutral leptons within the reach of FASER 2 [36,38] has recently been discussed in Ref. [20]. Although in this study we focus on collider searches for new physics, it is important to note that the KOTO anomaly can also have profound cosmological consequences that would have to be thoroughly investigated, especially if the observed excess persists, cf. Ref. [29] for a recent discussion.

Last but not least, FASER complementarity to BSM searches in kaon factories extends beyond the current anomaly. We have illustrated this for a simplified model with ALPs coupled to $S U(2)_{W}$ gauge bosons that dominantly decay to $\gamma \gamma$ pairs. FASER will probe regions of the parameter space relevant for several distinct searches for LLPs decaying either inside or outside the KOTO and NA62 detectors. It will, therefore, provide an independent probe of such scenarios. In a longer term, improved bounds can be obtained by FASER HL or FASER 2 detectors operating during the HL-LHC phase.

The upcoming run of the LHC will push the limits of intensity-frontier exploration of new physics to the next level. The FASER experiment will play a vital role in this endeavor toward potentially groundbreaking discoveries.

\section{ACKNOWLEDGMENTS}

We would like to thank Jonathan Feng, Sam Homiller, and Ben Lillard for useful discussions and comments on the 
manuscript. We are also grateful to the authors and maintainers of many open-source software packages, including CRMC [51], EPOS [50] Jupyter notebooks [71], MATPLOTLIB [72], NumPy [73], PYLHE [74], PYTHIA8 [58], and SCIKIT-HEP [75]. F. K. is supported by the Department of Energy under Grant No. DE-AC02-76SF00515. S. T. is supported by the Lancaster-Manchester-Sheffield Consortium for Fundamental Physics under STFC Grant No. ST/P000800/1. S. T. is partially supported by the Polish Ministry of Science and Higher Education through its scholarship for young and outstanding scientists (Decision No. 1190/E-78/STYP/14/2019).

\section{APPENDIX: MESON DECAY BRANCHING FRACTIONS TO DARK HIGGS BOSON AND ALP}

Below, we list the branching fractions of the dominant meson decay modes to produce light scalar and pseudoscalar particles in the models discussed in Secs. IV B, IV C, and V.

\section{Scalar decay boson}

For a light scalar, as defined in Eq. (7), the dominant production modes are rare decays of $\eta \mathrm{s}, \eta^{\prime} \mathrm{s}$, kaons, and $b$-quarks, with the following branching fractions adapted from Refs. [15,35] and references therein:

$$
\begin{gathered}
\mathcal{B}\left(K^{+} \rightarrow \pi^{+} X\right) \simeq 2.0 \times 10^{-3} \times \epsilon_{q}^{2} \lambda^{\frac{1}{2}}\left(m_{K}, m_{\pi}, m_{a}\right), \\
\mathcal{B}\left(K_{L} \rightarrow \pi^{0} X\right) \simeq 7.0 \times 10^{-3} \times \epsilon_{q}^{2} \lambda^{\frac{1}{2}}\left(m_{K}, m_{\pi}, m_{a}\right), \\
\mathcal{B}\left(K_{S} \rightarrow \pi^{0} X\right) \simeq 2.2 \times 10^{-6} \times \epsilon_{q}^{2} \lambda^{\frac{1}{2}}\left(m_{K}, m_{\pi}, m_{a}\right), \\
\mathcal{B}\left(\eta \rightarrow \pi^{0} X\right) \simeq 3.4 \times 10^{-5} \times \epsilon_{q}^{2} \lambda^{\frac{1}{2}}\left(m_{\eta}, m_{\pi}, m_{a}\right), \\
\mathcal{B}\left(\eta^{\prime} \rightarrow \eta X\right) \simeq 7.2 \times 10^{-5} \times \epsilon_{q}^{2} \lambda^{\frac{1}{2}}\left(m_{\eta^{\prime}}, m_{\eta}, m_{a}\right),
\end{gathered}
$$

$$
\mathcal{B}(b \rightarrow s X) \simeq 5.7 \times \epsilon_{q}^{2}\left(1-m_{X}^{2} / m_{b}^{2}\right)^{2},
$$

with

$$
\lambda\left(m_{1}, m_{2}, m_{3}\right)=\left[1-\frac{\left(m_{2}+m_{3}\right)^{2}}{m_{1}^{2}}\right]\left[1-\frac{\left(m_{2}-m_{3}\right)^{2}}{m_{1}^{2}}\right] .
$$

\section{ALP with the $\mathrm{SU}(2)_{\mathrm{W}}$ couplings}

For an ALP, as defined in Eq. (10) and with $g=g_{a W W} \cdot \mathrm{GeV}$, the relevant branching fractions for kaon decays read [25]

$$
\begin{aligned}
\mathcal{B}\left(K^{+} \rightarrow \pi^{+} a\right) & \simeq 10.5 \times g^{2} \lambda^{\frac{1}{2}}\left(m_{K}, m_{\pi}, m_{a}\right), \\
\mathcal{B}\left(K_{L} \rightarrow \pi^{0} a\right) & \simeq 4.5 \times g^{2} \lambda^{\frac{1}{2}}\left(m_{K}, m_{\pi}, m_{a}\right) .
\end{aligned}
$$

In the case of $B$-mesons, we follow the data-driven approach discussed in Ref. [67] and use $\mathcal{B}(b \rightarrow s a) \approx$ $5 \times\left[\mathcal{B}(B \rightarrow K a)+\mathcal{B}\left(B \rightarrow K^{*} a\right)\right]$ with the individual branching fractions taken from Ref. [66],

$$
\begin{aligned}
\mathcal{B}(B \rightarrow K a) & \simeq 2 \times 10^{4} \times g^{2} \lambda^{\frac{1}{2}}\left(m_{B}, m_{K}, m_{a}\right) F_{K}^{2}, \\
\mathcal{B}\left(B \rightarrow K^{*} a\right) & \simeq 2 \times 10^{4} \times g^{2} \lambda^{\frac{3}{2}}\left(m_{B}, m_{K^{*}}, m_{a}\right) F_{K^{*}}^{2},
\end{aligned}
$$

where the relevant form factors are given by $[76,77]$

$$
F_{K}=\frac{0.33}{1-m_{a}^{2} /\left(38 \mathrm{GeV}^{2}\right)}
$$

and

$$
F_{K^{*}}=\frac{1.35}{1-m_{a}^{2} /\left(28 \mathrm{GeV}^{2}\right)}-\frac{1}{1-m_{a}^{2} /\left(37 \mathrm{GeV}^{2}\right)} .
$$

[1] S. Shinohara, Search for the rare decay $K_{L} \rightarrow \pi^{0} \nu \bar{\nu}$ at J-PARC KOTO experiment, KAON 2019 (2019).

[2] T. Kitahara, T. Okui, G. Perez, Y. Soreq, and K. Tobioka, New Physics Implications of Recent Search for $K_{L} \rightarrow \pi^{0} \nu \bar{\nu}$ at KOTO, Phys. Rev. Lett. 124, 071801 (2020).

[3] J. L. Feng, I. Galon, F. Kling, and S. Trojanowski, ForwArd Search ExpeRiment at the LHC, Phys. Rev. D 97, 035001 (2018).

[4] A. Ariga et al. (FASER Collaboration), Letter of intent for FASER: ForwArd Search ExpeRiment at the LHC (to be published).
[5] A. Ariga et al. (FASER Collaboration), Technical Proposal for FASER: ForwArd Search ExpeRiment at the LHC (to be published).

[6] T. Yamanaka (KOTO Collaboration), The J-PARC KOTO experiment, Prog. Theor. Exp. Phys. 2012 (2012) $02 \mathrm{~B} 006$.

[7] S. Nagamiya, Introduction to J-PARC, Prog. Theor. Exp. Phys. 2012, 02B001 (2012).

[8] J. Ahn et al. (KOTO Collaboration), Search for the $K_{L} \rightarrow$ $\pi^{0} \nu \bar{\nu}$ and $K_{L} \rightarrow \pi^{0} X^{0}$ Decays at the J-PARC KOTO Experiment, Phys. Rev. Lett. 122, 021802 (2019). 
[9] A. J. Buras, D. Buttazzo, J. Girrbach-Noe, and R. Knegjens, $K^{+} \rightarrow \pi^{+} \nu \bar{\nu}$ and $K_{L} \rightarrow \pi^{0} \nu \bar{\nu}$ in the Standard Model: Status and perspectives, J. High Energy Phys. 11 (2015) 033.

[10] Y. Grossman and Y. Nir, $K_{L} \rightarrow \pi^{0} \nu \bar{\nu}$ beyond the standard model, Phys. Lett. B 398, 163 (1997).

[11] A. Artamonov et al. (E949 Collaboration), New Measurement of the $K^{+} \rightarrow \pi^{+} \nu \bar{\nu}$ Branching Ratio, Phys. Rev. Lett. 101, 191802 (2008).

[12] A. Artamonov et al. (BNL-E949 Collaboration), Study of the decay $K^{+} \rightarrow \pi^{+} \nu \bar{\nu}$ in the momentum region $140<P_{\pi}<199 \mathrm{MeV} / \mathrm{c}$, Phys. Rev. D 79, 092004 (2009).

[13] G. Ruggiero, New result on $K^{+} \rightarrow \pi^{+} \nu \bar{\nu}$ from the NA62 experiment, KAON2019, 2019.

[14] M. Fabbrichesi and E. Gabrielli, Dark-sector physics in the search for the rare decays $K^{+} \rightarrow \pi^{+} \bar{\nu} \nu$ and $K_{L} \rightarrow \pi^{0} \bar{\nu} \nu$, arXiv:1911.03755.

[15] D. Egana-Ugrinovic, S. Homiller, and P. Meade, Light Scalars and the KOTO Anomaly, Phys. Rev. Lett. 124, 191801 (2020).

[16] T. Li, X.-D. Ma, and M. A. Schmidt, Implication of $K \rightarrow \pi \nu \bar{\nu}$ for generic neutrino interactions in effective field theories, Phys. Rev. D 101, 055019 (2020).

[17] P. B. Dev, R. N. Mohapatra, and Y. Zhang, Constraints on long-lived light scalars with flavor-changing couplings and the KOTO anomaly, Phys. Rev. D 101, 075014 (2020).

[18] J. Liu, N. McGinnis, C. E. Wagner, and X.-P. Wang, A light scalar explanation of $(g-2)_{\mu}$ and the KOTO anomaly, J. High Energy Phys. 04 (2020) 197.

[19] Y. Jho, S. M. Lee, S. C. Park, Y. Park, and P.-Y. Tseng, Light gauge boson interpretation for $(g-2)_{\mu}$ and the $K_{L} \rightarrow$ $\pi^{0}+$ (invisible) anomaly at the J-PARC KOTO experiment, J. High Energy Phys. 04 (2020) 086.

[20] J. M. Cline, M. Puel, and T. Toma, A little theory of everything, with heavy neutral leptons, J. High Energy Phys. 05 (2020) 039.

[21] X.-G. He, X.-D. Ma, J. Tandean, and G. Valencia, Breaking the Grossman-Nir bound in kaon decays, J. High Energy Phys. 04 (2020) 057.

[22] R. Ziegler, J. Zupan, and R. Zwicky, Three exceptions to the Grossman-Nir bound, arXiv:2005.00451.

[23] Y. Liao, H.-L. Wang, C.-Y. Yao, and J. Zhang, An imprint of a new light particle at KOTO?, arXiv:2005.00753.

[24] X.-G. He, X.-D. Ma, J. Tandean, and G. Valencia, Evading the Grossman-Nir bound with $\Delta I=3 / 2$ new physics, arXiv:2005.02942.

[25] S. Gori, G. Perez, and K. Tobioka, KOTO vs. NA62 dark scalar searches, arXiv:2005.05170.

[26] M. Hostert, K. Kaneta, and M. Pospelov, Pair production of dark particles in meson decays, arXiv:2005.07102.

[27] A. Datta, S. Kamali, and D. Marfatia, Dark sector origin of the KOTO and MiniBooNE anomalies, arXiv:2005.08920.

[28] B. Dutta, S. Ghosh, and T. Li, Explaining $(g-2)_{\mu, e}$, KOTO anomaly and MinibooNE excess in an extended Higgs model with sterile neutrinos, arXiv:2006.01319.

[29] W. Altmannshofer, B. V. Lehmann, and S. Profumo, Cosmological implications of the KOTO excess, arXiv:2006 .05064 .

[30] X. Liu, Y. Li, T. Li, and B. Zhu, The light Sgoldstino phenomenology: Explanations for the muon $(g-2)$ deviation and KOTO anomaly, arXiv:2006.08869.
[31] P. Archer-Smith and Y. Zhang, Higgs portal from the atmosphere to hyper-K, arXiv:2005.08980.

[32] K. Fuyuto, W.-S. Hou, and M. Kohda, Loophole in $K \rightarrow \pi \nu \bar{\nu}$ Search and New Weak Leptonic Forces, Phys. Rev. Lett. 114, 171802 (2015).

[33] J. Beacham et al., Physics beyond colliders at CERN: Beyond the Standard Model working group report, J. Phys. G 47, 010501 (2020).

[34] J. Alimena et al., Searching for long-lived particles beyond the Standard Model at the large hadron collider, arXiv: 1903.04497.

[35] J. L. Feng, I. Galon, F. Kling, and S. Trojanowski, Dark Higgs bosons at the ForwArd Search ExpeRiment, Phys. Rev. D 97, 055034 (2018).

[36] F. Kling and S. Trojanowski, Heavy neutral leptons at FASER, Phys. Rev. D 97, 095016 (2018).

[37] J. L. Feng, I. Galon, F. Kling, and S. Trojanowski, Axionlike particles at FASER: The LHC as a photon beam dump, Phys. Rev. D 98, 055021 (2018).

[38] A. Ariga et al. (FASER Collaboration), FASERs physics reach for long-lived particles, Phys. Rev. D 99, 095011 (2019).

[39] A. Berlin and F. Kling, Inelastic Dark Matter at the LHC Lifetime Frontier: ATLAS, CMS, LHCb, CODEX-b, FASER, and MATHUSLA, Phys. Rev. D 99, 015021 (2019).

[40] A. Ariga et al. (FASER Collaboration), FASER: ForwArd Search ExpeRiment at the LHC, arXiv:1901.04468.

[41] K. Jodłowski, F. Kling, L. Roszkowski, and S. Trojanowski, Extending the reach of FASER, MATHUSLA, and SHiP towards smaller lifetimes using secondary particle production, Phys. Rev. D 101, 095020 (2020).

[42] H. Abreu et al. (FASER Collaboration), Detecting and studying high-energy collider neutrinos with FASER at the LHC, arXiv:1908.02310.

[43] H. Abreu et al. (FASER Collaboration), Technical proposal: FASERnu, arXiv:2001.03073.

[44] F. Kling, Probing light gauge bosons in tau neutrino experiments, arXiv:2005.03594.

[45] M. Tanabashi et al. (Particle Data Group), Review of particle physics, Phys. Rev. D 98, 030001 (2018).

[46] F. Bergsma et al. (CHARM Collaboration), Search for axion like particle production in $400-\mathrm{GeV}$ proton-copper interactions, Phys. Lett. 157B, 458 (1985).

[47] J. Blumlein et al., Limits on neutral light scalar and pseudoscalar particles in a proton beam dump experiment, Z. Phys. C 51, 341 (1991).

[48] J. Blumlein et al., Limits on the mass of light (pseudo)scalar particles from Bethe-Heitler $e^{+} e^{-}$and $\mu^{+} \mu^{-}$pair production in a proton-iron beam dump experiment, Int. J. Mod. Phys. A 07, 3835 (1992).

[49] E. Abouzaid et al. (KTeV Collaboration), Final results from the $\mathrm{KTeV}$ experiment on the decay $K_{L} \rightarrow \pi^{0} \gamma \gamma$, Phys. Rev. D 77, 112004 (2008).

[50] T. Pierog, I. Karpenko, J. Katzy, E. Yatsenko, and K. Werner, EPOS LHC: Test of collective hadronization with data measured at the CERN large hadron collider, Phys. Rev. C 92, 034906 (2015).

[51] C. Baus, T. Pierog, and R. Ulrich, Cosmic Ray Monte Carlo (CRMC), https://web.ikp.kit.edu/rulrich/crmc.html. 
[52] S. Alekhin et al., A facility to search for hidden particles at the CERN SPS: The SHiP physics case, Rep. Prog. Phys. 79, 124201 (2016).

[53] G. Bennett et al. (Muon g-2 Collaboration), Final report of the muon E821 anomalous magnetic moment measurement at BNL, Phys. Rev. D 73, 072003 (2006).

[54] J. Bjorken, S. Ecklund, W. Nelson, A. Abashian, C. Church, B. Lu, L. Mo, T. Nunamaker, and P. Rassmann, Search for neutral metastable penetrating particles produced in the SLAC beam dump, Phys. Rev. D 38, 3375 (1988).

[55] M. Davier and H. Nguyen Ngoc, An unambiguous search for a light Higgs boson, Phys. Lett. B 229, 150 (1989).

[56] A. Alavi-Harati et al. (KTeV Collaboration), Search for the Rare Decay $K_{L} \rightarrow \pi^{0} e^{+} e^{-}$, Phys. Rev. Lett. 93, 021805 (2004).

[57] T. Sjostrand, S. Mrenna, and P.Z. Skands, PYTHIA6.4 physics and manual, J. High Energy Phys. 05 (2006) 026.

[58] T. Sjstrand, S. Ask, J. R. Christiansen, R. Corke, N. Desai, P. Ilten, S. Mrenna, S. Prestel, C. O. Rasmussen, and P.Z. Skands, An introduction to PYTHIA8.2, Comput. Phys. Commun. 191, 159 (2015).

[59] I. Boiarska, K. Bondarenko, A. Boyarsky, V. Gorkavenko, M. Ovchynnikov, and A. Sokolenko, Phenomenology of GeV-scale scalar portal, J. High Energy Phys. 11 (2019) 162.

[60] V. V. Gligorov, S. Knapen, M. Papucci, and D. J. Robinson, Searching for long-lived particles: A compact detector for exotics at LHCb, Phys. Rev. D 97, 015023 (2018).

[61] D. Curtin et al., Long-lived particles at the energy frontier: The MATHUSLA physics case, Rep. Prog. Phys. 82, 116201 (2019).

[62] K. Babu, C. F. Kolda, and J. March-Russell, Implications of generalized $Z-Z^{\prime}$ mixing, Phys. Rev. D 57, 6788 (1998).

[63] H. Davoudiasl, H.-S. Lee, and W. J. Marciano, 'Dark' Z implications for parity violation, rare meson decays, and Higgs physics, Phys. Rev. D 85, 115019 (2012).

[64] J. A. Dror, R. Lasenby, and M. Pospelov, Light vectors coupled to bosonic currents, Phys. Rev. D 99, 055016 (2019).
[65] J. Grygier et al. (Belle Collaboration), Search for $\boldsymbol{B} \rightarrow \boldsymbol{h} \nu \overline{\boldsymbol{\nu}}$ decays with semileptonic tagging at Belle, Phys. Rev. D 96, 091101 (2017); 97, 099902(A) (2018).

[66] E. Izaguirre, T. Lin, and B. Shuve, Searching for Axionlike Particles in Flavor-Changing Neutral Current Processes, Phys. Rev. Lett. 118, 111802 (2017).

[67] D. Aloni, Y. Soreq, and M. Williams, Coupling QCD-Scale Axionlike Particles to Gluons, Phys. Rev. Lett. 123, 031803 (2019).

[68] M. Gavela, R. Houtz, P. Quilez, R. Del Rey, and O. Sumensari, Flavor constraints on electroweak ALP couplings, Eur. Phys. J. C 79, 369 (2019).

[69] M. J. Dolan, T. Ferber, C. Hearty, F. Kahlhoefer, and K. Schmidt-Hoberg, Revised constraints and Belle II sensitivity for visible and invisible axion-like particles, J. High Energy Phys. 12 (2017) 094.

[70] M. Bauer, M. Neubert, and A. Thamm, Collider probes of axion-like particles, J. High Energy Phys. 12 (2017) 044.

[71] T. Kluyver et al., Jupyter notebooks - A publishing format for reproducible computational workflows, in Positioning and Power in Academic Publishing: Players, Agents and Agendas (IOS Press, The Netherlands, 2016), pp. 87-90, https://eprints.soton.ac.uk/403913/.

[72] J. D. Hunter, MATPLOTLIB: A 2d graphics environment, Comput. Sci.\& Eng. 9, 90 (2007).

[73] T. Oliphant, NumPy: A guide to NumPy. USA: Trelgol publishing, 2006, http://www.numpy.org/.

[74] L. Heinrich, lukasheinrich/pylhe v0.0.4, 2018, https:// doi.org/10.5281/zenodo.1217032.

[75] E. Rodrigues, The Scikit-HEP project, EPJ Web Conf. 214, 06005 (2019).

[76] P. Ball and R. Zwicky, New results on $B \rightarrow \pi, K, \eta$ decay form-factors from light-cone sum rules, Phys. Rev. D 71, 014015 (2005).

[77] P. Ball and R. Zwicky, $B_{d, s} \rightarrow \rho, \omega, K^{*}, \phi$ decay formfactors from light-cone sum rules revisited, Phys. Rev. D 71, 014029 (2005). 